\title{
Individual and work related factors associated with symptoms of musculoskeletal complaints. I A quantitative registration system
}

\author{
R H Westgaard, T Jansen
}

\begin{abstract}
A registration system for symptoms of occupational musculoskeletal injuries has been developed and evaluated. The system is based on the recording of two aspects of the pain sensation: the intensity (five point scale) and the frequency (three point scale) of pain. The two primary indicators are summed to give a combined symptom score. The scoring system was evaluated in terms of its ability to predict the probability of a medical consultation due to a musculoskeletal pain problem. The intensity and frequency parameters were shown to contribute significantly and to carry equal weight when optimising the symptom score by a logistic regression analysis. In the scoring of pain symptoms a distinction was made between pain experienced during and outside working hours and only the first category was included. The separation eliminated some symptoms that the workers did not consider work related (mostly due to migraine, arthritis). Repeated interviews of 14 subjects showed, however, that this procedure could cause classification problems.
\end{abstract}

The common manifestation of an occupational musculoskeletal injury is a pain syndrome, which may or may not be accompanied by more objective signs of an injury. It is often difficult to establish definite medical diagnoses of the underlying complaint ${ }^{1-3}$ and the problem may therefore be assessed by quantifying the pain syndrome itself.

Division of Organisation and Work Science, the Norwegian Institute of Technology, Trondheim, Norway

R H Westgaard

National Institute of Occupational Health, Department of Physiology, PO Box 8149 Dep, Oslo 1, Norway

$T$ Jansen
The lack of objective methods for the measurement of pain has always hampered research into pain related illnesses, as considerable doubt regarding the reliability and validity of subjective pain estimates is often expressed. Various methods for the measurement of physiological parameters indicative of pain have been developed, ${ }^{4}$ but such methods are only applicable to pain experienced at the time of pain measurement. Alternatively, in depth information of pain experiences may be obtained by large questionnaires such as the McGill pain questionnaire. ${ }^{56}$

The requirement of a standardised method for the measurement of occupational pain was recognised by Nordic scientists who developed questionnaires for the registration of symptoms of pain at various body locations. ${ }^{7}$ Extended questionnaires were developed for the neck and shoulders and the lumbar region. The questionnaires provided a basis for calculating the point prevalence and period prevalence of pain symptoms by establishing the occurrence of symptoms during the past seven days and the past year. In the extended questionnaires the respondent also indicated the number of days with pain, whether he had visited a doctor, and whether the problem had caused a reduction in normal activities during the past 12 months. The Nordic questionnaires were formulated with an emphasis on simplicity to allow completion without supervision by health personnel. They have the important advantage of standardisation, which allows comparison of epidemiological data from different studies, but they provide limited information about pain qualities, pain intensities, and pain patterns.

In a study of individual and work related factors influencing muscle pain among sewing machine operators, quantitative information on pain levels was needed, but the detailed information on pain modalities in the large clinical questionnaires was considered excessive. A questionnaire that focused on the intensity and pattern of symptoms, intended to be used in structured interviews, was therefore developed. This paper presents the questionnaire and the relation between the reported pain symptoms 
Table 1 Intensity of discomfort

\begin{tabular}{ll}
\hline Value & Explanatory text \\
\hline 0 & $\begin{array}{l}\text { No complaints } \\
\text { Almost no complaints; only slight feeling of } \\
\text { discomfort at breaks when not concentrating on } \\
\text { the work task }\end{array}$ \\
2 & $\begin{array}{l}\text { Slight, but noticeable complaints when performing } \\
\text { the work tasks. However, these are of sufficiently } \\
\text { low intensity not to interfere with your } \\
\text { performance at work }\end{array}$ \\
Relatively strong complaints during work, making \\
it necessary to maintain a conscious effort in order \\
to carry out the work task. It is necessary at times \\
to have breaks due to the discomfort experienced.
\end{tabular}

and behavioural manifestations of pain. A report, presenting preliminary results of the study, has been published. ${ }^{8}$

\section{Methods}

SYMPTOM QUESTIONNAIRE

The questionnaire was designed to obtain information on symptoms of pain or discomfort during the time of employment in the current work situation for each of eight body regions: head, neck, shoulders and upper arms, lower arms (including elbow) and hands, the lumbar region of the back, hips and buttock, thighs, and knees and ankles. Symptoms in shoulders, arms, thighs, and knees and ankles were indicated as left, right, or bilateral. The questionnaire was intended to be used by a skilled professional, usually a medical doctor, when interviewing the respondent. The placement of symptoms at different body regions was made by the doctor in the course of the interview.

The subject had to indicate intensity and frequency of symptoms. Symptom intensity was indicated on a five-point scale with anchoring text provided for the five levels (table 1). The intensity scale was designed to cover problems graded from very slight to ones where virtually everyone would respond with a medical consultation and a sick leave. The anchoring text was therefore formulated in

Table 2 Duration of discomfort

\begin{tabular}{lll}
\hline Value & $\begin{array}{l}\text { Short } \\
\text { anchor }\end{array}$ & Explanatory text \\
\hline 0 & Monthly & $\begin{array}{l}\text { Rare complaints occurring on a monthly } \\
\text { basis, but not frequently enough to be } \\
\text { considered weekly }\end{array}$ \\
1 & Weekly & $\begin{array}{l}\text { The complaints are occurring one or several } \\
\text { days each week over a period of at least two } \\
\text { months } \\
\text { The complaints are occurring every working } \\
\text { day over a period of at least two weeks }\end{array}$ \\
\hline
\end{tabular}

terms of the ability to carry out work while enduring the pain. The subjects were allowed to use half points if they felt that their problem was best described as an intensity level somewhere between two of the anchoring texts.

Thereafter, the subject chose one of three values to indicate the frequency of the discomfort when experiencing the episode of highest pain or discomfort intensity during the period of employment. The frequency was indicated as daily, weekly, or infrequent (table 2). Specific information regarding the occurrence of symptoms was recorded. Finally, the subject indicated whether the situation had deteriorated, improved, or remained stable after the symptoms first appeared.

The two scores of intensity and frequency of symptoms were combined in a symptom score by adding the two related values. The symptom score ranged from 0 (no complaint) to 6 (daily occurrence of severe symptoms for at least two weeks), the last considered to represent an extreme value for subjects carrying out regular work duties. The scoring system allowed a complaint at an intermediate symptom level to be reached by different combinations of frequency and intensity scores. Thus, a symptom level of 4 may indicate daily occurrence of complaints of moderate intensity, weekly occurrence of relatively significant complaints, or rare occurrence of very severe complaints.

\section{MATERIAL}

A total of 210 female sewing machine operators at four separate locations in Norway, and a group of 31 office and four laboratory workers in the same company were interviewed. One sewing machine operator refused to participate. Data on sick leave were collected and local muscle load was quantified by electromyograph (EMG) recordings for a smaller number of subjects. ${ }^{910}$ The interview was repeated two months later for 14 subjects.

The interview preceded an extensive medical examination, the results of which are to be published in a separate paper. The interview and medical examinations lasted on average one hour and 20 minutes, and they were all carried out by a consultant of physical medicine (TJ). The subjects were questioned on working conditions, life situation, and the use of medical services before responding to the symptom questionnaire. ${ }^{11}$ Information about medical consultations, usually with the company doctor, was verified by inspection of the medical records.

\section{STATISTICS}

To determine whether a weighting of the intensity or frequency scores was appropriate when combining the two scores in a symptom score, a multivariate logistic regression analysis was performed with 
medical consultation due to musculoskeletal pain (yes/no) as the dependent variable and the highest symptom and frequency scores, regardless of body location, as the independent variables. The analysis was then repeated twice; firstly, with the restriction that only subjects where the two scores referred to the same body region were included and secondly, as above, but only including subjects with the two scores referring to the shoulder and neck region.

The correlation between symptom score and time from the medical consultation to the interview was evaluated by linear regression analysis. The statistical analyses were performed with the SAS statistical package.

\section{Results}

SCORING OF INTENSITY AND FREQUENCY OF SYMPTOMS A total of 1960 indications of pain or discomfort at different body locations was made by the 245 subjects. About half of these were different from zero (1037 scores, $53 \%$ ). The first point of interest was to examine the assumption that the subjects were able to comprehend frequency and intensity of pain as independent parameters.

The three frequency scores were used to almost the same extent with 346,330 , and 361 indications of infrequent, weekly, or daily occurrence respectively. The intensity values mostly used were $2,2 \cdot 5$, and 3 , representing almost $90 \%$ of the positive intensity scores. This indicates that the subjects differentiated on symptom intensity within the middle range of scores in table 1, but that they did not distinguish between no pain at all and "just noticeable" pain as defined in table 1. At the high end of the scale most subjects would probably respond with a medical consultation and sick leave before experiencing pain symptoms of such intensity.

Figure 1A shows the percentage distribution of intensity scores for the three possible frequency scores of symptoms. The intensity scores associated with each frequency score is represented by one of three bars, summating to $100 \%$ over the range of intensity scores. The intensity distribution was similar for the three frequency scores, but with a tendency of a positive correlation betwen the two scores. Some correlation between the two symptom parameters is not unexpected.

Figure 1B shows the related intensity and frequency scores added to give a combined "symptom score." The rationale for this transformation is to achieve a data reduction retaining the symptoms of pain or discomfort on a single scale that reflects both symptom qualities. The different shading of the columns indicates different combinations of intensity and frequency scores. The symptom scores show a wide distribution, mainly between values 2 and 5 . The low end of the scale is dominated by infrequent
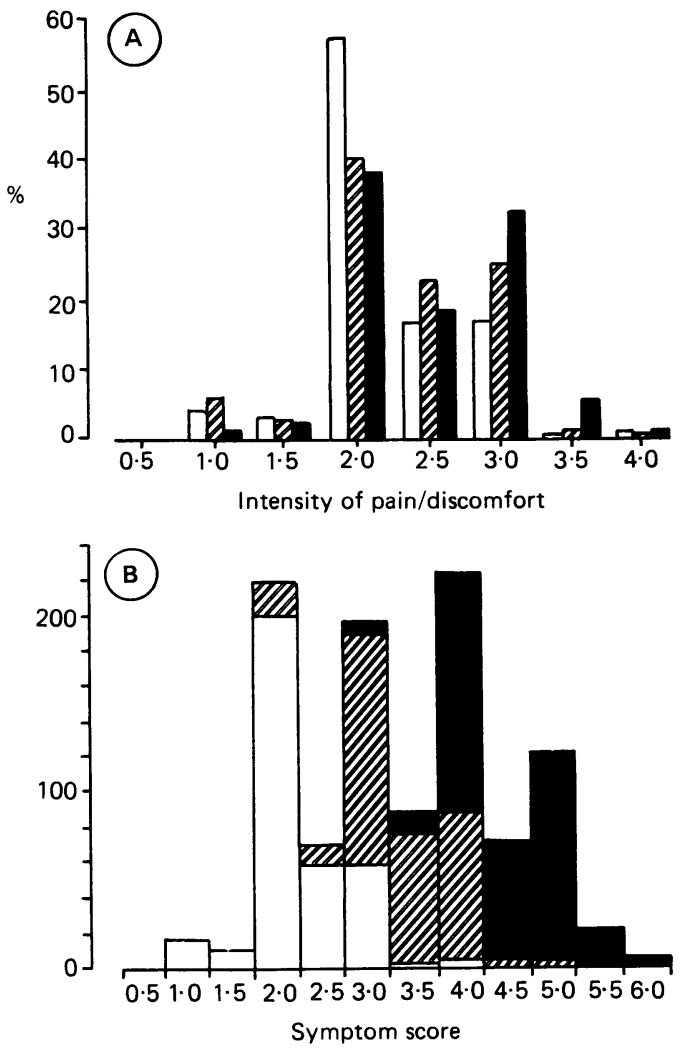

Figure 1 (A) Distribution of positive intensity scores for infrequent (open columns), weekly (hatched columns), and daily (filled columns) frequency scores. Each set of columns adds up to $100 \%$. (B) Distribution of positive symptom scores. Columns show symptom scores based on infrequent (open part of columns), weekly (hatched part of columns), and daily (filled part of columns) frequency of symptoms.

symptoms of different intensities (open sections of columns) and values of 4.5 or higher by daily symptoms (filled sections of columns). Symptom levels 3 and 4 mainly consist of two combinations of frequency and intensity scores.

\section{SYMPTOM LEVEL AND BEHAVIOURAL EFFECTS}

The anchoring text of the intensity scale was phrased in terms of behavioural responses at work. Information on medical consultations due to symptoms of pain was also obtained, and used as a basis for normalisation of the symptom scores. The correlation between the two sets of parameters indicated at what degree of reported pain a risk of initiating a medical consultation and possibly a sick leave for this group of workers existed. Particularly, it was of interest to know how the two primary scores (intensity and frequency) and the combined symptom score were able to predict this response, and whether 
Table 3 Relation between intensity and frequency of musculoskeletal symptoms and medical consultations

\begin{tabular}{|c|c|c|c|c|c|c|c|}
\hline & \multicolumn{4}{|c|}{ Intensity of symptoms } & \multicolumn{3}{|c|}{ Frequency of symptoms } \\
\hline & 0 & 2 & 3 & 4 & 0 & 1 & 2 \\
\hline \multirow{2}{*}{$\begin{array}{l}\text { No of workers } \\
\text { Medical consultations (\%) } \\
\text { due to symptoms }\end{array}$} & 6 & 54 & 157 & 28 & 36 & 57 & 151 \\
\hline & 0 & 44 & 73 & 96 & 25 & 49 & 85 \\
\hline
\end{tabular}

information on both parameters improved the predictive power.

Table 3 shows the number of workers with different intensity and frequency scores (highest value regardless of body location), and the percentage with a medical consultation. For the intensity scale half values were grouped together with the higher full value, and the subject with a highest intensity score of 1 was included with those without pain symptoms. The fraction of workers with a medical consultation increased with increasing severity of the complaint on both scales, as expected. A $3 \times 3$ cross table with the number of workers and the percentage with a medical consultation for low, medium, and high intensity and frequency scores was constructed (table 4). Mostly, a monotonic increase in fraction of workers with medical consultation with increasing severity of both parameters was found. The fraction recording a medical consultation appears somewhat high in the cell with medium intensity and low frequency of the symptom $(58 \%)$, but this value (and that of other cells) is based on a low number of subjects.

A logistic regression analysis was then performed, with medical consultation due to musculoskeletal pain symptoms as the dependent parameter, and the highest intensity and symptom levels, regardless of body location, as independent parameters (table 5). A preliminary analysis confirmed that no interaction between the two primary parameters existed. The weighting coefficients for highest intensity and highest frequency of pain were equal $\left(A_{1}=1.01, A_{2}=\right.$ 0.95 ) indicating that both parameters contribute significantly to an estimator of the probability of a medical consultation due to musculoskeletal pain.

Table 4 Medical consultations for musculoskeletal pain as a function of intensity and frequency of symptomst

\begin{tabular}{llll}
\hline & \multicolumn{3}{l}{ Frequency } \\
\cline { 2 - 4 } Intensity & low (0) & medium (1) & high (2) \\
\hline low & 24 & 13 & 23 \\
$(0-2)$ & $8 \%$ & $30 \%$ & $78 \%$ \\
medium & 12 & 38 & 107 \\
$(2 \cdot 5-3)$ & $58 \%$ & $50 \%$ & $82 \%$ \\
high & - & 6 & 22 \\
$(3 \cdot 5-4)$ & - & $83 \%$ & $100 \%$ \\
\hline
\end{tabular}

The number of workers with the appropriate combination of intensity and frequency scores (highest values independent of body location), and the percentage of workers with a medical consultation due to such symptoms are indicated.
When limiting the analysis to subjects in which the highest intensity and the highest frequency scores referred to the same body region, the coefficients were unchanged $\left(A_{1}=1.05, A_{2}=0.95\right)$.

The above analyses assume that the body location of the pain symptoms does not influence the probability of initiating a medical consultation. The analysis was therefore repeated for subjects where the highest symptom level referred to the shoulder and neck region. This included most workers who had recorded a medical consultation. The restriction did not change the result $\left(A_{1}=1 \cdot 2, A_{2}=1 \cdot 3, p=\right.$ 0.024 and 0.001 respectively).

Figure 2A shows the highest symptom score regardless of body location for all subjects. The shaded areas indicate, for each symptom level, the number of subjects who have recorded a medical consultation due to pain symptoms. The fraction of workers with a visit to a doctor because of musculoskeletal complaints is indicated by the fully drawn line in fig 2 B. All workers with symptom levels of 5.5 and 6 have consulted a medical doctor because of this problem, reducing to $11 \%$ at a symptom score of 2 . The relatively high fraction of $63 \%$ at a symptom score of 2.5 is based on only eight subjects who all referred to consultations more than a year before the interview.

Figure 3 shows the highest symptom level at the time of the medical consultation as a function of time from the consultation to the interview. The reported pain level showed a clear tendency to reduce with an increase in time since the consultation. The two workers indicating a symptom level of 2 referred to visits almost four years before the interview, whereas those indicating a symptom level of 2.5 visited the doctor one to five years before the interview. The records with symptom level 3 were also dominated by long periods. At the high end of the symptom scale

Table 5 Logistic regression analysis

\begin{tabular}{lcl}
\hline Coefficient & Estimated value $(95 \% \mathrm{CI})$ & p Value \\
\hline $\mathrm{A}_{0}$ & $-2.87(-4.68$ to -1.05$)$ & \\
$\mathrm{A}_{1}$ & $1.01(0.30-1.71)$ & 0.009 \\
$\mathrm{~A}_{2}$ & $0.95(0.45-1.41)$ & 0.0004 \\
\hline
\end{tabular}

Logistic regression analysis of highest pain intensity and frequency scores as predictors of probability of a medical consultation. $A_{0}$ to $A_{2}$ are the regression coefficients of the analysis; $A_{1}$ and $A_{2}$ are the weighting coefficients of the highest intensity and highest frequency scores. 

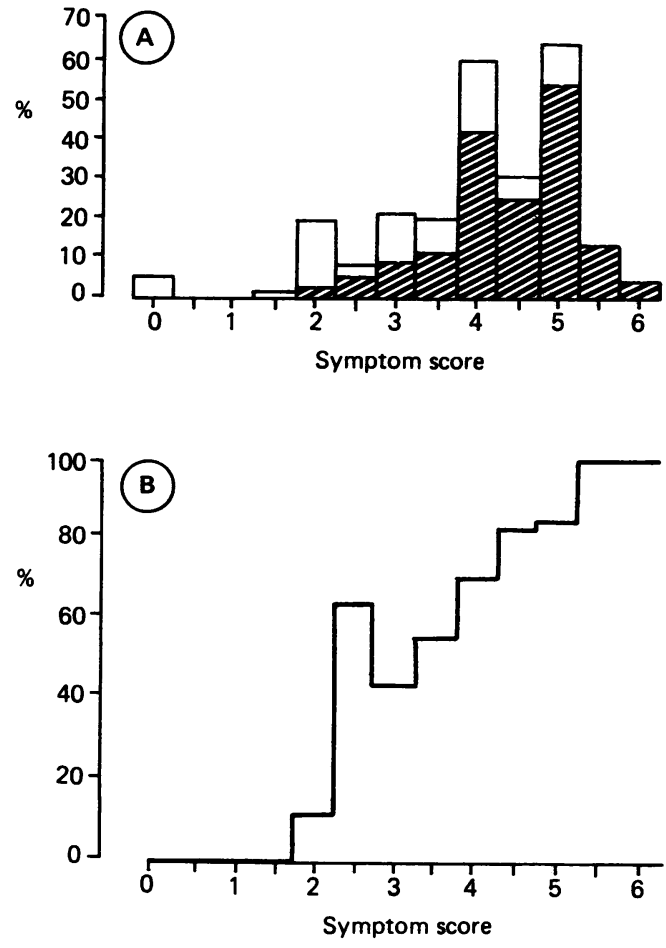

Figure 2 (A) Distribution of highest symptom level, regardless of body location, for 245 production and office workers. Hatched part of columns indicates workers with medical consultations due to musculoskeletal pain. (B) \% of workers with medical consultations due to musculoskeletal pain as a function of symptom level

symptom values of 5.5 and 6 had an equally clear tendency to be associated with recent visits to a doctor. The regression coefficient of the line in fig 3 is $0 \cdot 15$, significantly different from zero $(p<0.001)$. The mean symptom level at the time of visit to a doctor was 4.6. If the three values referring to consultations more than seven years ago were excluded, the regression coefficient increased to $0 \cdot 21$.

No significant difference was found in the regression analysis when only workers with medical consultations followed by a subsequent sick leave were included. Nor were any differences found between geographically separated groups of sewing machine operators performing similar work tasks, or between different body locations with the highest symptom score. Office workers reported significantly lower symptom levels, however, than the production workers at the time of the medical consultation (mean values $4 \cdot 0$ and $4 \cdot 7, p=0.01$ ).

RELIABILITY OF THE SYMPTOM SCORE

To obtain an indication of the reliability of the symptom scores, the interview was repeated two months later for 14 subjects, resulting in 112 indica- tions of symptom level at different body locations. Fifty seven of these $(50 \%)$ scored within 0.5 units of the first value and $33(29 \%)$ deviated by two units or more, pointing towards low reliability of the symptom score. Examination of the accompanying notes established that a major reason for the discrepancy was a change in bias, and that symptoms predominantly not experienced at work were excluded in the first interview and included in the second (11 cases). Also, several subjects had experienced episodes of pain between the two interviews (nine cases). Other reasons for discrepancies were more extensive localisation of symptoms (low back pain spreading to hips, four cases), infrequent symptoms only remembered in one interview (five cases), and unexplained discrepancies (four cases). Thus at least 20 of the large deviations in symptom score were not primarily due to poor reliability and the scoring system appeared to have the potential of becoming very reliable. This was verified in later studies when a difference of $2-3 \%$ in responses of one scale point and $1 \%$ of two scale points was found for the intensity scale. ${ }^{12}$ Similar results were obtained for the frequency scale.

In the further analysis of the material from the first interview ${ }^{11}$ symptoms due to problems mainly occurring outside working hours were eliminated. Symptoms experienced at work were always included, even when the subject considered these to be due to factors independent of the working conditions.

\section{Discussion}

The main differences between the pain questionnaire of this paper and the Nordic questionnaire, ${ }^{7}$ which both aim to quantify symptoms of occupational musculoskeletal complaints, are (1) the attempt to quantify the intensity of pain in terms of behavioural responses at work, (2) to quantify the occurrence (frequency) of pain when it is experienced rather than

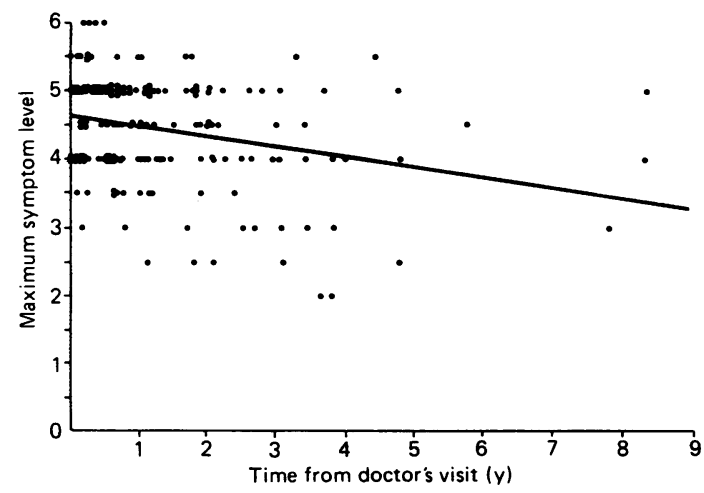

Figure 3 Highest symptom level at time of last medical consultation due to musculoskeletal problems as a function of time from the medical consultation to the interview. 
the number of days with painful experiences in the past year, and (3) to derive a new parameter, the symptom score, as the sum of the two primary parameters. It should be noted that a different weighting of the two parameters, up to a factor of four, may be appropriate in view of the confidence intervals of the two coefficients. The main point of importance, however, is that the knowledge of both parameters improves the prediction of the risk of a medical consultation. The absence of weighting also retains the simplicity of the scoring system.

The symptom score was compared with information on medical consultations. This was considered a better choice for the normalisation analysis than the sick leave statistics, as it reflects the subjects' own opinions of their health state, whereas sick leave is one of several possible outcomes of the consultation. Seventy six per cent of the production workers with a medical consultation recorded a subsequent sick leave. ${ }^{11}$ Alternative outcomes are treatment with pain relieving drugs, or physical therapy, or both without sick leave. No indication of different symptom scores at the time of the medical consultation for workers with and without a subsequent sick leave was found.

The time dependent change in symptom score at the time of medical consultation, depending on the time elapsed from the consultation to the interview, may be due to a time dependent attenuation in the recollection of pain symptoms. This hypothesis is not easily verified, but the correlation between reported pain symptoms and the rate of medical consultations would be skewed towards higher symptom levels if only workers with recent experience of pain symptoms were considered. Thus most production workers with a recent medical consultation indicated symptom levels of four or higher.

Alternative scoring systems for musculoskeletal pain symptoms were not considered. In particular, a scoring of the time dimension based on the number of days with symptoms, as in the Nordic questionnaire, is an alternative to the frequency score of the present study and should be evaluated with regard to predictive power for medical consultations. Also, if a need exists for a clinical description of the pain problem, alternative questionnaires are available. ${ }^{13}$

The symptom data may be dichotomised if a simplified data treatment is wanted. Data would then be presented as yes/no results, but with the added information that the branch point represents a level where, for instance, $50 \%$ of the subjects with this symptom level are likely to seek medical advice for their complaints. With symptom frequencies as high as 70 to $80 \%$ for the shoulder neck region, however, a continuous scoring parameter is more likely to show differences in symptom levels than frequency data, which is more common in epidemiological research.

The experience with the first version of the questionnaire emphasised the need for careful planning of the interview to achieve an acceptable degree of reliability. A distinction between pain experienced at and outside work was made, with only the first category included in the response. Pain predominantly experienced outside working hours was usually located in body regions with a low level of strain at work, and was associated with ailments which the workers did not consider work related (migraine, arthritis). The separation of pain experienced at and outside work therefore appeared to focus possible work related pain symptoms. The system for classification of symptoms must be well defined, however, and the necessary information must be readily available. It should therefore be considered if the quality of information allows this distinction to be made.

It was also necessary to delineate the different body segments carefully, which is probably best done by a graphic illustration similar to that used by the Nordic questionnaire. This illustration (and the Nordic questionnaire) was not available at the time of the interviews. The scheme for separation of body segments is easily modified. For instance, if a particular problem occurs with the upper extremeities, the arm may be subdivided into several regions. If it is not necessary to distinguish adjacent body regions, improved reliability can be achieved by considering these as one region.

A problem with the first version of the interview was the lack of a definite time for the measurement of symptom level, preventing the calculation of a valid period prevalence. This is easily corrected by including a question on symptoms during the past 12 months. Symptoms located in the head must also be handled carefuly. Those included as pain symptoms in this study were described as tension headache, localised to the dorsal aspects of the head.

Finally, it should be noted that many factors may influence the correlation between symptom level and the need for medical advice. The degree of pain symptoms that initiate a medical consultation will vary between subjects, depending on factors such as pain tolerance, job motivation, and social situation. In this study office workers appeared to have a lower threshold for using this service than the production workers. The availability and cost of medical service would have an effect, as may company policies towards their workers and broader cultural issues. If a similar scoring system was used to characterise occupational musculoskeletal pain experienced by workers in another social setting, a normalisation analysis to correlate pain symptoms with medical consultations should also be performed for that material.

We acknowledge the support of employees and management of Helly-Hansen A/S in carrying out this project. Mr Leiv Sandvik provided helpful 
advice on the use of statistical techniques.

Requests for reprints to: $\mathrm{R} \mathrm{H} \mathrm{Westgaard,} \mathrm{Division} \mathrm{of}$ Organisation and Work Science, the Norwegian Institute of Technology, Trondheim, Norway.

1 Simons DG. Muscle pain syndromes. American Journal of Physical Medicine 1976;55:15-42.

2 Waris P. Occupational cervicobrachial syndromes. Scand J Work Environ Health 1980;6 (suppl 3):3-14.

3 Hagberg $M$. Occupational musculoskeletal stress and disorders of the neck and shoulder: a review of possible pathophysiology. Int Arch Occup Environ Health 1984;53:269-78.

4 Bromm B. Pain measurement in man. Amsterdam: Elsevier, 1984.

5 Melzack $R$. The McGill pain questionnaire: major properties and scoring methods. Pain 1975;1:277-99.

6 Melzack R. Measurement of the dimensions of pain experience. In: Bromm B, ed. Pain measurement in man. Amsterdam: Elsevier, 1984:327-48.

7 Kuorinka I, Jonsson B, Kilbom A, Vinterberg H, BieringSørensen F, Andersson G, Jørgensen K. Standardised Nordic questionnaires for the analysis of musculoskeletal symptoms. Applied Ergonomics 1987;18:233-7.

8 Westgaard RH, Wærsted M, Jansen T, Korsund K. Loads and load-related complaints of production workers at HellyHansen Inc. Oslo: National Institute of Occupational Health, 1984. (In Norwegian.)

9 Westgaard RH, Wærsted M, Jansen T, Aarả A. Muscle load and illness associated with constrained body postures. In: Corlett N, Wilson J, Manenica I, eds. The ergonomics of working postures. London: Taylor and Francis, 1986:5-18.

10 Warsted M, Westgaard RH. Working hours as a risk factor in the development of musculoskeletal complaints. Ergonomics 1991;34:265-276.

11 Westgaard $\mathrm{RH}$, Jansen $\mathrm{T}$. Individual and work related factors associated with symptoms of musculoskeletal complaints. II Different risk factors among sewing machine operators. $\mathrm{Br} J$ Ind Med 1992;49:154-62.

12 Aarås A. Load-related musculo-skeletal illness: is ergonomic workplace design a sufficient remedy? In: Haslegrave CM, Wilson JR, Corlett EN, Manenica I, eds. London: Taylor and Francis, Work design in practice. 1990:30-40.

13 Chapman CR, Casey KL, Dubner R, Foley KM, Gracely RH, Reading AE. Pain measurement: an overview. Pain 1985;22: 1-31.

Accepted 17 June 1991 